\title{
A Closer Look at Texture Metrics for Visualization
}

\author{
Haleh Hagh-Shenas ${ }^{*} \quad$ Victoria Interrante Cheong-Hee Park \\ Computer Science Department, University of Minnesota, Minneapolis, MN, 55455
}

\begin{abstract}
This paper presents some insights into perceptual metrics for texture pattern categorization. An increasing number of researchers in the field of visualization are trying to exploit texture patterns to overcome the innate limitations of three dimensional color spaces. However, a comprehensive understanding of the most important features by which people group textures is essential for effective texture utilization in visualization. There have been a number of studies aiming at finding the perceptual dimensions of the texture. However, in order to use texture for multivariate visualization we need to first realize the circumstances under which each of these classification holds. In this paper we discuss the results of our three recent studies intended to gain greater insight into perceptual texture metrics. The first and second experiments investigate the role that orientation, scale and contrast play in characterizing a texture pattern. The third experiment is designed to understand the perceptual rules people utilize in arranging texture patterns based on the perceived directionality. Finally, in our last section we present our current effort in designing a computational method which orders the input textures based on directionality and explain its correlation with the human study.
\end{abstract}

Keywords: Texture metrics, filtering, Texture classification, Visualization

\section{INTRODUCTION}

One of the constant challenges in the field of visualization is constructing methods to display large and complex datasets in an effective and flexible manner. Examples of such datasets are geographical, environmental, and medical data. Having an enormous range of detail in color and pattern at the same time, textures seem to have vast potentials to be used as a flexible tool in visualization. By matching various data features with different perceptual texture metrics, researchers can convey more information at the same location and hence expand viewer's understanding of the underlying physical phenomenon being visualized. If we can communicate more information to the observers in a reliable and effective manner, we are able to help researchers from different fields in real time detection of patterns or anomalies.

It is apparent, however, that before the full potentials of textures for visualization can be realized, we need to completely understand the most important features by which people group textures. In the first part of this paper we present our most recent experiments designed to distinguish differences between the set of visual features that people might use for texture classification under different time constraints. We particularly wanted to know how subjects would react to differences of scale, rotation, and contrast of each texture and how these results can be reconsolidated with Ware and Knight's [Ware and Knight 92] texture features: scale, orientation and contrast. Our hypothesis was that orientation, scale, and contrast are useful manipulable features "within" a particular single texture pattern but that "between" different texture patterns it is other visual features that we are at times relying on to group/classify the textures.

In our first study, for each texture that Rao and Lohse [Rao and Lohse 93a] used, we added versions that differed from the original in scale, contrast, and rotation. Subjects who participated in the study were asked to cluster textures together, thus replicating Rao and Lohse's study. We also designed a computerized experiment to determine if different texture metrics were used under different time constraints. After showing four images for a period of time, subjects were asked to group the images either horizontally or vertically into two groups of two. The duration in which the subjects viewed the images was one second in one experiment, and without limit in another. Anova analysis was applied to the results of the computerized experiment.

\footnotetext{
*e-mail: haleh@cs.umn.edu
} 
In the Second half of the paper we present a user study which was designed to better understand the way people arrange textures based on perceived directionality and compare its results with our computational method by computing Kendall concordance factor and spearman correlation coefficient.

Section 1.1 summarizes previous work on finding texture dimensions. Section 2 consists of two major subsections. They describe the texture metric user studies we conducted and their corresponding results in addition to discussing the possible explanations for the obtained results. Section 3 explains our study of directionality of the texture and discusses our computational method and compares it with our user study on directionality. Finally, the last section presents a brief conclusion.

\subsection{Background}

Recently, researchers in computer graphics and visualization have started to use textures in multivariate visualization. Healy and Enns [Healy and Enns 99] present a method in which the combination of simple texture patterns and color is used to visualize multivariate data elements. They show that using texture dimensions and perceptually uniform colors can increase the number of attribute values that are needed to be displayed simultaneously. In a similar study Kirby et al. [Kirby et al 99], used a mixture of discrete and continuous visual elements and combined them in layers using artistic guidance in flow visualization.

In terms of finding the perceptual texture metrics Julesz' [Julesz 81] early research identifies primitive dimensions of textures such as brightness and orientation. Treisman and Gormican's [Treisman and Gormican 88] experiments concluded that only a small number of features such as size, contrast and curvature are extracted in early vision. Among the pioneering works is also Tamura et. al.'s [Tamura et al 89] research which identified contrast, coarseness and directionality as the most significant texture dimensions. Liu and Picard [Liu and Picard 96] presented an image model based on 2-D Wold decomposition of homogeneous random fields and defined their texture metrics as: periodicity, directionality and randomness.

In their paper, Ware and Knight [Ware and Knight 92] explain how studies in vision research help advance the information visualization field. Although it is still not clear how human visual system processes spatial information, the vision studies suggest that it uses parallel arrays of neurons whose functionality can be modeled by Gabor functions. Based on the Gabor model, Ware and Knight argue that the primary texture dimensions in the context of human perception are orientation, size and contrast. In separate studies, Rao and Lohse [Rao and Lohse 93b], [Rao and Lohse 93a] identified repetitive vs. non-repetitive, high contrast and non-directional vs. low-contrast and directional; granular, coarse and low-complexity vs. non-granular fine and high-complexity as the three most significant dimensions of texture. However, to our knowledge none of the above studies have investigated the effect of having different transformations of same textures present, nor have they studied the possible differences among the pre-attentive metrics and features people use when they have no time constraint. Our target transformations in the first section of the paper are scale, rotation, and contrast and in the second section we focus on directionality in particular.

\section{EXPERIMENTS ON TEXTURE METRIC}

We performed three experiments. The first and third involved actual prints of Brodatz textures and the second one was a computerized experiment consisting of two parts. The first study was designed to observe the general reaction of subjects to the presence of transformed (scaled or rotated or contrasted) versions of each texture in relation to other similar textures. In the first two experiments, subjects were asked to cluster textures and in the third one were asked to arrange the textures based on the perceived directionality

The goal of the computerized experiment was to identify the pre-attentive features used for texture classification. In short the goal of the first two experiments was to decide if scale, contrast and orientation could explain all the classification rules people apply to textures. The first study is presented in Section 3.1, and the second one in section 3.2 and the last one is presented in Section 3.3. The computerized method for finding the directionality is followed in Section 4. 


\subsection{Experiment 1}

\subsubsection{Subjects}

Five subjects with various areas of specialization participated in this study. Their Ages ranged from 25 to 50 . They were not given any criteria for judging the similarity of textures that they were instructed to cluster. Furthermore, they had complete freedom in the number of clusters and the way they wanted to cluster the textures.

\subsubsection{Texture Selection}

We decided to use the same thirty Brodatz textures Rao and Lohse [Rao and Lohse 93] used in their experiment as our starting set. In addition, we constructed three transformed sets of all textures in Adobe's Photoshop. Overall, four sets of thirty images were used. The first set was the original Brodatz textures. The second set was the $50 \%$ scaled down version of the original Brodatz textures. Textures in the third set were all forty-five degree rotations of the original Brodatz textures. In the fourth set, we used the curve tool to manipulate the contrast of each image. The same patch size as the original texture was cut from each transformed version. However, a different part of each texture was cut from the four images to prevent subjects from picture matching. It should be noted that the changes in contrast were dramatic, mostly resulting in the black and white images that were faithful to the original in form but had lost the color scheme.

The above process resulted in one hundred and twenty patches which were positioned randomly on a table. Some examples of textures used in the study along with their transformed versions, can be seen in Figure 1.

\subsubsection{Task}

Replicating Rao and Lohse' [RL93a] procedure, we chose a bottom-up sorting task to emphasize the similarity between our patterns and to be able to create hierarchical groups for similar textures. We did not constrain our subjects to use any specific criteria nor did we constrain the amount of time they could spend thinking. The subjects were asked to first look at all the textures and then group them to as many clusters as they desired based on any features they perceived as similar. The experimenter did not give any information to the participants as to the process by which they were supposed to choose the texture groups. The task typically took half an hour to an hour. After the first grouping, the subjects were asked about their reasons for making each group and the main features that brought the group members together. Then, the subjects were asked to merge some of their groups that they perceived as being similar in another feature until they were no longer comfortable with further hierarchical merging. Their reflections on all these grouping were noted by the experimenter.

\subsubsection{Experimental Results}

The first reaction of four out of five participants was to find each texture and the three corresponding transformations and group them together. The only few exceptions happened when subjects were not able to find the matching textures of the contrasted versions because the contrasted versions were noisy and at times hard to identify what they were. They mostly called this group the unidentified textures. After much contemplation between grouping the textures by form or by contrast, the $5^{\text {th }}$ subject decided to group rotated and scaled versions of a texture with itself but set the high contrast images aside into their own group. Such categorization suggested that although scale, orientation and contrast as stated by Ware and Knight [Ware and Knight 92] might be reliable texture features when clustering different textures, they were not the best choice when a texture and transformed copies of itself were present. Throughout the clustering process, all the five subjects brought up the question of the higher level meaning of the texture.
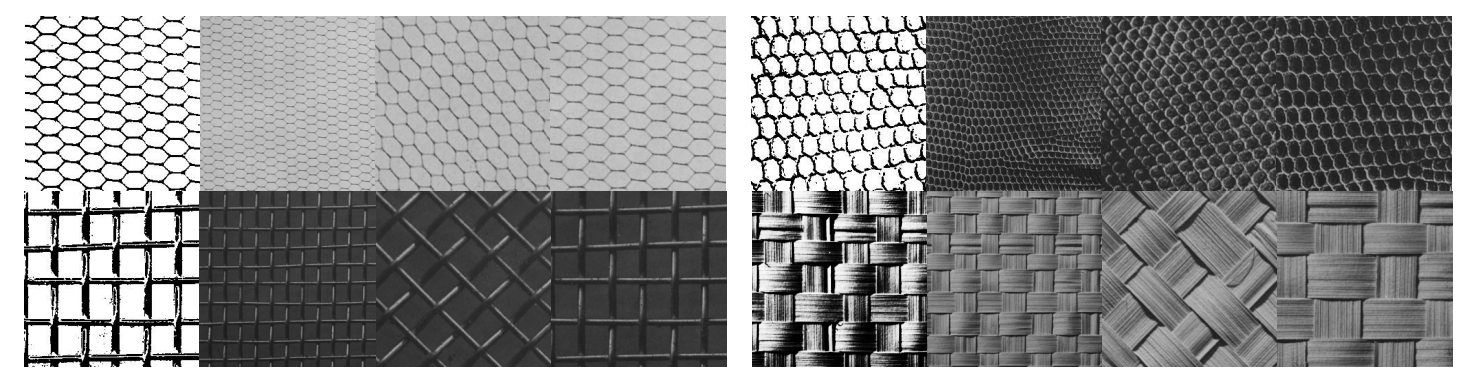

Figure 1: Examples of contrasted, scaled (down to 50\%) and rotated versions of textures used in the first study. The original textures in Brodatz from left to right, top to bottom are: D34, D3, D1 and D64. 
For example during the merging process some subjects wanted to know if pattern D37 was wood or water; if they decided it was wood, they wanted to categorize it with other wood patterns (D71 and D69) and if not with other directional textures such as D93. Another interesting example was pattern D86. Most subjects perceived it as cement wall or floor and regrouped it with bricks. Such grouping shows that at times the conceptual meaning of textures could override other texture features like orientation, scale and contrast. These results led to our decision for conducting a separate experiment where people were asked to make classification decisions within strictly limited times. Specifically, we wanted to know if minimizing the time people had to "contemplate" and "understand" the images had any worsening effects on their ability to consider these types of higher level considerations.

\subsection{Experiment 2}

\subsubsection{Subjects}

Seventeen subjects with various areas of specialties participated in this study. Their Ages ranged from 20 to 30 . The subjects were naive to the purpose of the experiment throughout both parts and were not given any criteria for judging the similarity of textures that they were instructed to cluster.

\subsubsection{Texture Selection}

We had a total number of twenty one independent trials in this experiment; each trial consisted of four textures. These textures were a subcategory of the textures Rao and Lohse [Rao and Lohse 93a] used in their experiments plus a few others from the Brodatz album. This category consisted of the patterns that had some form of orientation or were comprised of repetitive elements capable of being scaled. Each of the twenty one groups consisted of a texture, a transformed version of that texture, a similar texture chosen by taking into consideration Rao and Lohse' grouping, and the same texture transformed by the same transformation. Each group was used as a seed trial. We had ten trials involving scaling textures down to $50 \%$ or $25 \%$ of their actual size, six involving rotation for 45 degree and five involving changes in contrast. Some of these seed trials can be seen in Figure 2.

\section{Part 1}

Eight repetitions of each seed trial were constructed, representing eight different layouts. Each layout was repeated twice so we had 336 actual trials $(21 \times 16)$ for part 1 . In order to give subjects some time to adjust and form conceptual rules, we repeated the first layout of all seed trials once before the actual sixteen repetitions were shown. The results of these twenty one trials were discarded.

Trials were shown to the subjects for the duration of one second each. In the following page, they were asked to choose between a vertical line (meaning they grouped the two textures on the right together and chose the two textures on the left as another cluster) and a horizontal one (i.e., the top patterns formed one cluster and the bottom patterns another cluster). Throughout this paper we call a trial's result a same-texture choice, if in that trial the subject had clustered a texture with transformed version of itself.

\section{Part 2}

In part 2 we used the same twenty one seed trials but repeated each of the eight layouts only once (hence we had 168 trials) and subjects were able to take as much time as they found necessary looking at each trial images. Subjects performed part 2 of the experiment immediately after finishing part 1. Classification page was also similar to part 1.

\subsubsection{Experimental Results}

Figure 3 shows the overall summary of the results that we found in part 1 and part 2 of our computerized experiment. The chart presents the average percentage of times textures were categorized with scaled/rotated/contrasted versions of themselves as opposed to going with a similar texture which was closer to them in scale, orientation, or contrast.

We computed the average percentages that a same-texture choice was made for each image set and each subject and calculated stdev and mean of these average values across subjects, transformation type, etc. Then, we calculated stderr measure as:

Stderr $=$ stdev/sqrt $(\#$ means_compared $)$

and used that to define the sizes of error bars in the charts. As Figure 3 shows, the average number of times textures were matched with the transformed version of themselves despite the differences in scale, orientation, or contrast was considerably higher in the second part, when people were able to spend more time examining the four textures in each trial. This suggests that scale, orientation, and contrast might be pre-attentive in nature and other high level laws guide the classification process when it is not required to be decided in real time. We used statistical software package 'MacAnova', developed at the Department of Statistics of University of Minnesota and by Oehlert. We performed a three-way, within subjects mixed analysis of variance (ANOVA) to evaluate the statistical significance of the results. We found significant main effects of subject $(\mathrm{p}=2.806 \mathrm{e}-12, \mathrm{~F}=25.47)$ and time $(\mathrm{p}=0.0005428, \mathrm{~F}=18.55)$, and a significant two-way interaction between subject and time $(\mathrm{p}=2.971 \mathrm{e}-12, \mathrm{~F}=6.358)$. We did not find a significant statistical significance of the results. We found significant main effects of subject $(\mathrm{p}=2.806 \mathrm{e}-12, \mathrm{~F}=25.47)$ and time $(\mathrm{p}=0.0005428, \mathrm{~F}=18.55)$, 
and a significant two-way interaction between subject and time $(\mathrm{p}=2.971 \mathrm{e}-12, \mathrm{~F}=6.358)$. We did not find a significant main effect of the type of transformation $(\mathrm{p}=0.4063, \mathrm{~F}=0.9266)$ nor a significant interaction between subject and transformation type $(\mathrm{p}=0.9195$, $\mathrm{F}=0.6723)$ or time and transformation type $(\mathrm{p}=0.2657, \mathrm{~F}=1.382)$.

We used Tukey's HSD ("Honestly Significant Difference") method to perform post-hoc pairwise comparisons of the average number of times people made a same-texture choice under the different time constraints. We found that the following differences were statistically significant: at the 0.05 level: people were $12 \%$ more likely to match a texture with itself when given unlimited time rather than just one second.

\subsection{Discussion}

It appears from the results of our experiment that there are small but significant differences in the extent people use scale, contrast and orientation as basis for texture categorization in preattentive tasks vs. the circumstances under which they have enough time for processing higher order metrics. These differences have been made apparent by including the same-texture transformations in our experiment, in addition to having different textures possessing qualities such as orientation and element size. As it was mentioned before, subjects seemed to apply different strategies for classification in part one and part two of the experiment: overall their texture categorization was more involving scale, orientation and contrast metrics in part1 vs. more same-texture classification in part2). One unexpected result was that we did not find any significant differences between the results corresponding to trials involving texture orientation, scale or contrast. One reason could be that we didn't have the same number of trials involving these transformations (As mentioned before, in our seed trials we had 10 trials with differences in scale, 6 with differences in orientation and five with differences in contrast). Complicating the matter is that it's possible that differences between two texture types included in each trial, was more salient in seed trials with one type of transformation than others (i.e. differences in rotation were at times more salient than contrast or scale). One other avenue for future work would be investigating the effect of different scale ratios (e.g. 50\% and $75 \%$ in our experiment). In our future experiments we are planning to address the above and similar issues. We also would like to design other experiments to investigate different sets of texture dimensions such as periodicity, directionality, randomness using a similar paradigm.

\section{DIRECTIONALITY}

\subsection{Human Perception}

The goal of this study was to investigate the methods by which people determine the ordering of a set of natural texture images based on the perceived "directionality" in the pattern. We also developed a computer program which attempts to determine this ordering computationally. We hope that the findings of the user study can help us to refine and improve our computational model of directionality perception.

\subsubsection{Subjects}

For this preliminary user study, we had eight subjects with various area of specialization. Their ages ranged from $25-30$.

\subsubsection{Texture Selection}

Sixty Eight textures from the Brodatz album were selected and one patch of 256x256 pixels of each texture was cut. Some of the textures had to be scaled down to include enough information about the image. An example of such textures is the swinging light (D43).

\subsubsection{Task}

Subjects were asked to arrange the textures on top of a table on which an axis showing the numbers from 1 to 90 was presented. Two labels, one at axis number 1 reading "Highly directional" and one at 90 reading "Non directional'" was also presented. A written instruction page asked the participant to carefully examine the images and arrange them against the axis based on the perceived directionality. This way each texture ended up with one number per subject such that the value of the number showed how directional that subject perceived the texture compared to others. Subjects were able to assign the same position (and hence the same number) to more than one texture. The texture samples were given to the subjects in a randomized pile. No other information was provided regarding the task nor was any time limit for completing it. The result will follow the section on the computational method. 

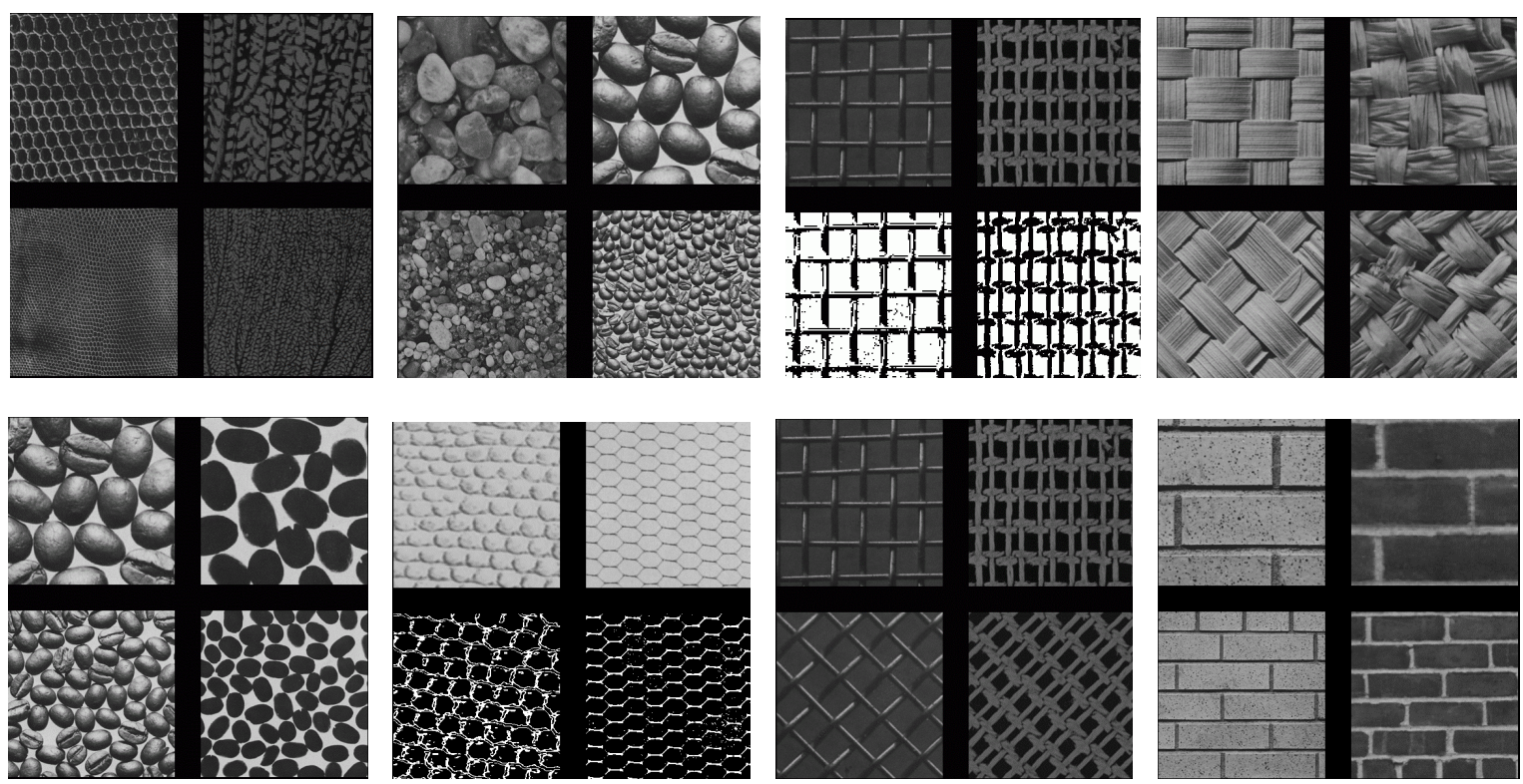

Figure 2: Examples of our texture grouping for part1 and part2 of the experiment 2

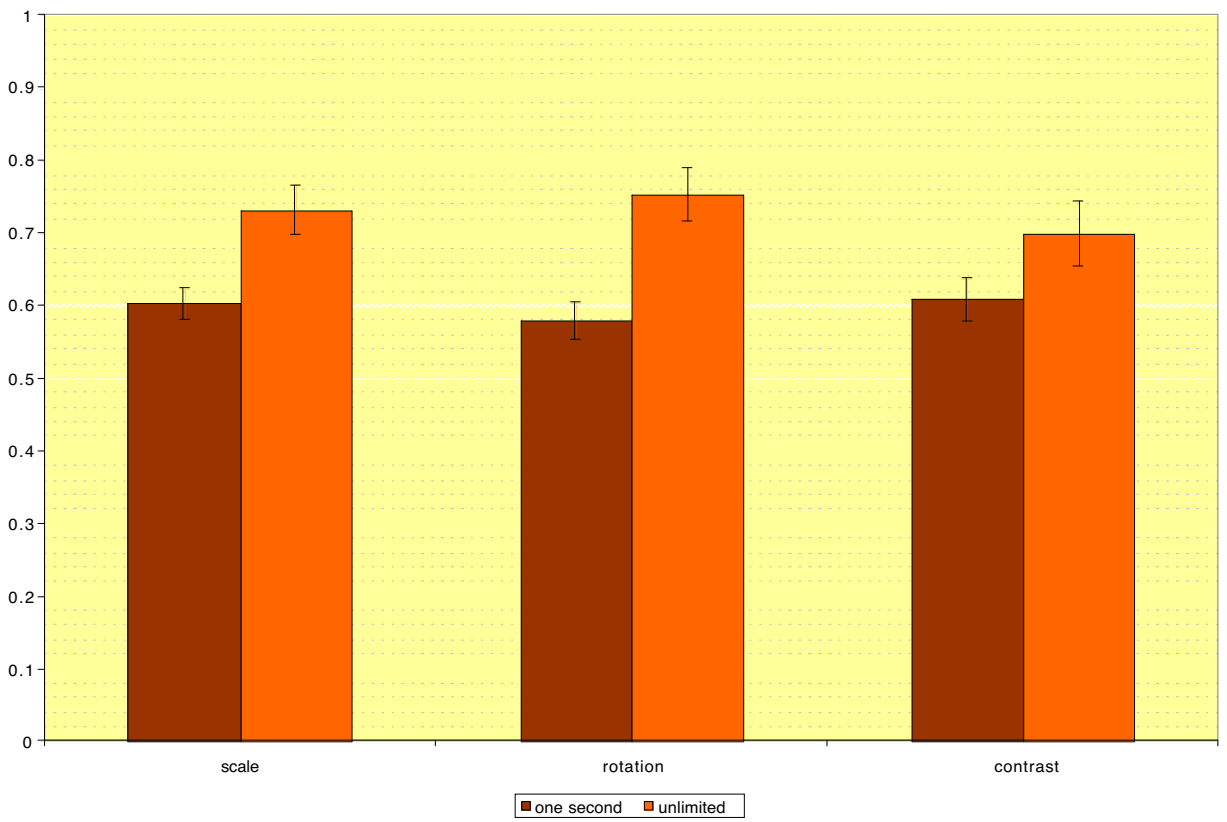

Figure 3: Average number of same-texture choices across all subject and trials for part1 (series 1, darker, when subjects had one second to see each pairs) and part2 (series2, lighter, when they had unlimited time). 


\subsection{Directionality: Computational Method}

Despite the great progress made so far in partitioning natural texture space there are still many more questions in regard to texture classifications to answer. For example we still don't know if it is possible to characterize the relevant natural textures' dimensions mathematically and interpolate between these metrics to fill out a texture space. Researchers have so far applied different methods to investigate the perceptual features of textures. Examples of such methods are gray level spatial dependency, co-occurrence based method, parametric models and power spectrum decomposition [Randen and Husoy 99, Ojala et. al. 96, Weszka et. al. 76, Liu and Picard 96]. In this section we investigate the potentials of Fourier Transform to analyze the perceptual features of our texture patterns and to classify them into meaningful categories based on directionality.

Throughout this section we consider a frequency domain in which the DC point is moved to the center. We have chosen the spectral decomposition analysis as our main method here because it can potentially deal with both structural textures over various scales as well as statistical textures. Furthermore, the invertiblity of DFT is a desirable factor which makes it possible to create the in-between textures from their frequency domain information.

To reduce the effect of aliasing in Fourier transform we first applied the Hanning window to all the images. A Gaussian filter then smoothed the DFT magnitude of these images and then the rectangular grid was converted to a polar coordinate.

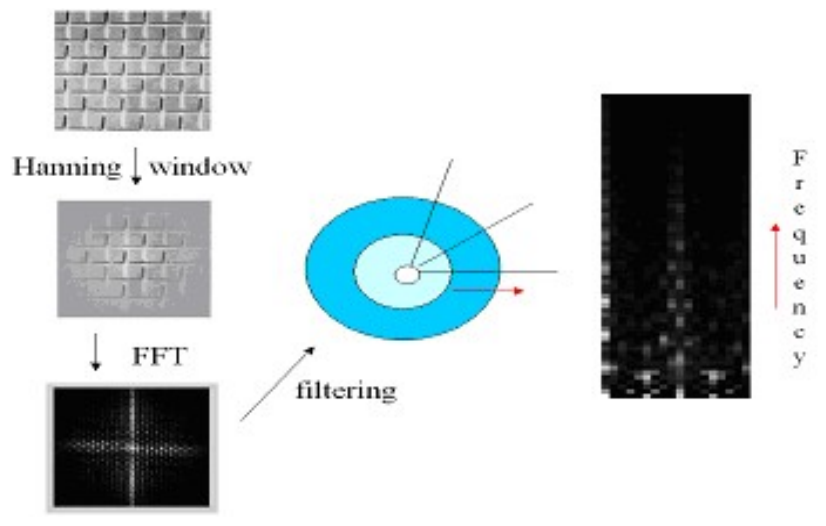

Figure 4: Steps for building Discrete Fourier Polar-coordinate matrix

The same textures which our participant in experiment 3 arranged based on directionality were scaled down to $128 \times 128$ and were used in this section. Because the magnitude space of DFT is symmetric, a frequency domain was divided by 18 lines through the DC point with 10 degrees interval from downward vertical line and 64 circles with maximum radius from the DC point. Based on this information an 18x64 matrix was built in which each row corresponded to a circle and each column to a line through the DC point in the frequency domain. We call this matrix a DFPM (Discrete Fourier Polar Coordinates Matrix).

\subsubsection{Directionality dimension}

We have applied this method to classify textures based on scale and contrast metrics and for structural grouping as well as directionality. Due to shortage of space we only present results concerning directionality which is the focus of our experiment 3. The closest study to our investigation is perhaps Tamura's work in which he conducted two kinds of experiments to compare six perceptual features over 16 textures: one based on the psychophysical judgment of Human subjects through pair comparison method and the other one based on a computational experiment. He measured the directionality by using local horizontal and vertical directional operators over texture intensity domain. However, to our knowledge no one has investigated the directionality metric over the broad range of textures we have used in this experiment. Liu and Picard decomposed the DFT domain into three components periodicity, directionality and 
randomness and utilized it for image retrieval. Although the agreement of their method's ranking of repetitiveness was tested through a human study, to our knowledge, no similar study on directionality was reported. Here we measure directionality effectively from DFPM by assigning a quantitative number to it for a broad range of textures. We also show that there is s strong agreement between the preliminary human experiment and the computer result.

Let $\mathrm{f}(\mathrm{i})$ be the sum of cells in the ith column of DFPM. The texture has a dominant direction at an angle $10^{*}$ maximum value at $i$ and the directionality is defined as

$$
\frac{\sum_{i=1}^{K}((\max f-f(i))}{K *(\max f)}
$$

where $\mathrm{K}$ is the number of column. A sample result can be seen in Figure 5 and the complete averaging in Appendix 1 and 2.

\subsubsection{Analysis and Result}

Some statistical methods were applied to the data. The goal was to decide if computer and Human results were statistically correlated and to find out how significant this correlation was. The Spearman rank correlation coefficient $r_{s}$ was calculated to assess the correlation between the computerized ranking and the average human ranking. Because at this point we felt that we didn't have enough number of human subjects we computed Kendall concordance coefficient W [Kendall and Gibbons 90] to make sure that there was overall agreement among our eight subjects and that the averaged ranking was reliable. The Euclidean arrangement of each subject was converted to a statistical ranking first (hence each texture had a number from 1-68 per each subject) and the spearman rank correlation coefficient was calculated to measure the degree of correlation. This measure is a number between -1 and 1 ; 1 showing complete agreement and -1 showing complete disagreement. This value for the averaged human ranking was 0.81 with $t=$ 13.85. The probability of error is then less than $0.1 \%(\mathrm{p}<0.001)$ meaning that the human and computer ranking are significantly correlated.

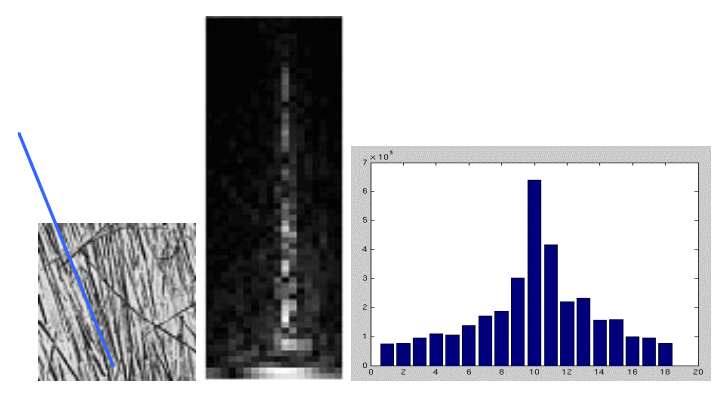

Figure 5: An example of a texture (D15) and its DFPM

Furthermore, the Kendall concordance coefficient $\mathrm{W}$ was measured at $0.80 \mathrm{~W}$ is generally a value between 0 and 1 : the value is 1 when subjects are in perfect agreement. The significance of this value was tested by calculating the approximation of $\chi^{2}$ [Kendall and Gibbons 90] which was computed to be 423.72 under the null hypothesis that there was no agreement among the rankings of different subjects. The probability for this value to occur under the null hypothesis is $\mathrm{p}<0.001$. Therefore the hypothesis can be rejected with the probability of error less than $0.1 \%$. In conclusion it seems like there is an underlying agreement among the criteria that each subject has used for his/her arrangement.

\section{CONCLUSION AND FUTURE WORK}

In this paper we have shown that at least under the presence of same-texture transformations, there are other high-level metrics that could override perceptual texture metrics discussed in earlier studies. In particular, we have shown that scale, orientation and contrast metrics tend to be used more often in pre-attentive tasks and they seem to interplay a more complicated role while there is time to ponder about texture similarities. 
In the sections on directionality we showed our effort toward designing a computational method which could order textures based on directionality while being perceptually in agreement with the human arrangement.

In our future work we plan to run more subjects for our directionality experiments to reach a more accurate estimate of a "perceptual ranking" based on direction and guided by these results tune our computational method to better represent it. We also plan to investigate any possible differences among male and female participants and also fully investigate the potentials of using DFPM by applying it to other texture metrics

We hope that by experiments such as ours which is directed at better understating of various texture dimensions, we are able to help researchers in computer graphics and visualization community to build perceptually efficient and reliable tools. To achieve this goal we feel there is a dire need for carrying on more investigations on texture understanding and eventually defining computational methods for texture synthesis. Such texture synthesis algorithms are able to match any data to the best possible candidates from among the texture space; a choice that also matches perceptual texture metrics.

\section{REFERENCES}

1. Healy C. and Enns J.: Large Datasets at a glance: Combining Textures and Colors in Scientific Visualization. IEEE transactions on visualization and computer graphics, VOL 5, NO 2 APRIL-JUNE (1999).

2. Julesz, B.: Textons, the elements of texture perception, and their interactions. Nature 290 (1981), 91-97.

3. Kendall M. and J. D. Gibbons J. D. Rank Correlation Methods. Oxford University Press, fifth edition, 1990.

4. Kirby, R.M., Marmanis, D.H. Laidlaw, D.H. .:Visualizing Multivalued Data from 2D Incompressible Flows Using Concepts from painting. IEEE Visualization, (1999).

5. Liu F., Picard R. .: Periodicity, Directionality and Randomness: Wold Features for Image Modeling and Retrieval. IEEE Transactions on Pattern Analysis and Machine Intelligence, (1996).

6. Ojala T. , Pietikainen M. and Harwood D., A comparative study of texture measures with classification based on feature distributions, pattern recognition, 29, 1996, 51-59.

7. Randen T., Husoy J., Filtering for texture classification: a comparative study, IEEE trans. On pattern analysis and machine intelligence, vol. 21(4), 1999.

8. Rao, A. R., and Lohse, G. L. .: Identifying high level features of texture perception. CVGIP: Graphics Models and Image Processing 55, 3 (1993), 218-233.

9. Rao, A. R., and Lohse, G. L.: Towards a texture naming system: Identifying relevant dimensions of texture. In Proceedings Visualization '93 (San Jose, California, 1993), pp. 220-227.

10. Tamura, H., Mori, S. and Yamawaki T. .: Textural features corresponding to textural properties, IEEE Trans. Systems Man Cybernet. 19, (1989) 1264-1274.

11. Treisman A. and S Gormican S. .: Feature analysis in early vision: Evidence from search asymmetries, Psych. Rev, 95(1), (1988), 15-48

12. Ware, C., Knight, W. .: Orderable dimensions of visual texture for data display: orientation, size, and contrast. ACM conference on Human Factors in Computing System. (CHI) (1992), pp 203-209.

13. Weszka J., Dyer C. and Rosenfeld A., A comparative study of texture measures for terrain classification, IEEE trans. Syst., Man, Cybern., SMC-6, Apr. 1976, 269-285. 
Appendix 1: Experiment 3, computerized ordering, numbers associated to each texture, the higher the number the higher the directionality. The next page, presents the average ranking of our participants for the first 42 textures which have some directionality present in the image. From top to bottom the directionality decreases and the right columns precedes the left column. The number on the left hand side of each texture presents the computational ordering for that particular texture.

\begin{tabular}{|l|r|r|r|r|}
\hline d049.pgm, & 0.913073 & & d007.pgm, & 0.488904 \\
\hline d025.pgm, & 0.84515 & & d018.pgm, & 0.472994 \\
\hline d053.pgm, & 0.81701 & & d093.pgm, & 0.454114 \\
\hline d021.pgm, & 0.816551 & & d010.pgm, & 0.449163 \\
\hline d095.pgm, & 0.765012 & & d005.pgm, & 0.447996 \\
\hline d001.pgm, & 0.751547 & & d071.pgm, & 0.419426 \\
\hline d047.pgm, & 0.749389 & & d019.pgm, & 0.406802 \\
\hline d096.pgm, & 0.748833 & & d012.pgm, & 0.406542 \\
\hline d020.pgm, & 0.747715 & & d084.pgm, & 0.40593 \\
\hline d015.pgm, & 0.730348 & & d041.pgm, & 0.360297 \\
\hline d083.pgm, & 0.726783 & & d088.pgm, & 0.339338 \\
\hline d006.pgm, & 0.719548 & & d082.pgm, & 0.33507 \\
\hline d068.pgm, & 0.707234 & & d074.pgm, & 0.333821 \\
\hline d072.pgm, & 0.695568 & & d057.pgm, & 0.329388 \\
\hline d076.pgm, & 0.692592 & & d091.pgm, & 0.313882 \\
\hline d094.pgm, & 0.691949 & & d063.pgm, & 0.302882 \\
\hline d037.pgm, & 0.663369 & & d045.pgm, & 0.301637 \\
\hline d046.pgm, & 0.659099 & & d043.pgm, & 0.299376 \\
\hline d034.pgm, & 0.656601 & & d087.pgm, & 0.298621 \\
\hline d65.pgm, & 0.656105 & & d024.pgm, & 0.296715 \\
\hline d085.pgm, & 0.639687 & & d112.pgm, & 0.279304 \\
\hline d101.pgm, & 0.634983 & & d028.pgm, & 0.26951 \\
\hline d077.pgm, & 0.631404 & & d002.pgm, & 0.264343 \\
\hline d064.pgm, & 0.624686 & & d110.pgm, & 0.260145 \\
\hline d016.pgm, & 0.621772 & & d062.pgm, & 0.25181 \\
\hline d035.pgm, & 0.614807 & & d030.pgm, & 0.248039 \\
\hline d055.pgm, & 0.610778 & & d111.pgm, & 0.229711 \\
\hline d052.pgm, & 0.573254 & & d027.pgm, & 0.212302 \\
\hline d003.pgm, & 0.56259 & & d097.pgm, & 0.2074 \\
\hline d081.pgm, & 0.547032 & & d075.pgm, & 0.204924 \\
\hline d103.pgm & 0.539387 & & d066.pgm, & 0.203139 \\
\hline d017.pgm, & 0.535827 & & d009.pgm, & 0.202524 \\
\hline d011.pgm, & 0.528326 & & d060.pgm, & 0.166816 \\
\hline d056.pgm, & 0.513575 & & d058.pgm, & 0.159155 \\
\hline
\end{tabular}



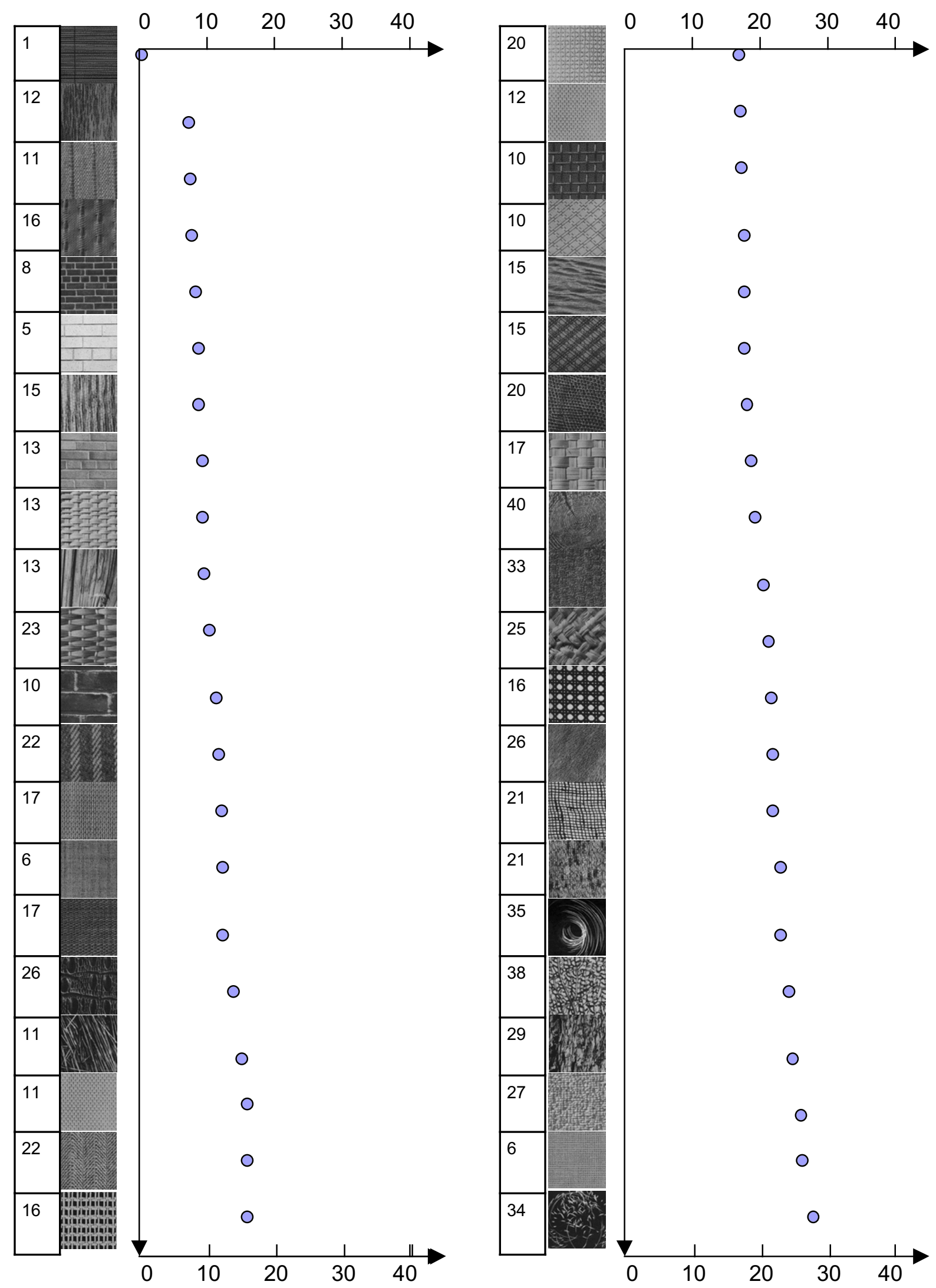